Table 1. Demographic and clinical characteristics at the time of inclusion and at 5 years.

\begin{tabular}{lccc}
\hline & $\begin{array}{c}\text { T0 } \\
(\mathbf{n}=83)\end{array}$ & $\begin{array}{c}\text { T5 } \\
(\mathbf{n}=83)\end{array}$ & $p$ \\
\hline Age years, mean \pm SD & $56.0 \pm 8.9$ & & - \\
Female, $\mathrm{n}(\%)$ & $75(90.4)$ & & - \\
Disease duration years median (q25-q75) & $10.4(4.4-15.7)$ & & - \\
CVRF, $\mathbf{n}$ (\%) & & & \\
Hypertension & $27(32.5)$ & $38(45.8)$ & $\mathbf{0 . 0 0 1}$ \\
Dyslipidemia & $25(30.1)$ & $16(19.3)$ & $\mathbf{0 . 0 4 1}$ \\
DM & $6(7.2)$ & $14(16.9)$ & $\mathbf{0 . 0 0 8}$ \\
Active smoking & $8(9.6)$ & $6(7.2)$ & $\mathrm{NS}$ \\
Overweight/Obesity & $67(81.7)$ & $63(81.9)$ & $\mathrm{NS}$ \\
Stroke & 0 & $4(4.8)$ & - \\
Clinical characteristics & & & \\
BMI kg/m ${ }^{2}$, mean \pm SD & $28.1 \pm 4.1$ & $28.5 \pm 4.3$ & $\mathrm{NS}$ \\
SBP mmHg, mean \pm SD & $123.7 \pm 18.4$ & $126.9 \pm 16.8$ & $\mathrm{NS}$ \\
Total cholesterol mg/dl, mean \pm SD & $180.8 \pm 29.0$ & $173.9 \pm 34.2$ & $\mathrm{NS}$ \\
Triglycerides mg/dl, mean \pm SD & $136.1 \pm 56.1$ & $146.1 \pm 64.2$ & $\mathrm{NS}$ \\
HDL-C mg/dl, mean \pm SD & $55.2 \pm 16.3$ & $55.1 \pm 15.9$ & $\mathrm{NS}$ \\
Statins, $\mathrm{n}(\%)$ & $9(10.8)$ & $13(15.7)$ & $\mathrm{NS}$ \\
Methotrexate, $\mathrm{n}$ (\%) & $71(85.5)$ & $63(75.9)$ & $\mathbf{0 . 0 3 9}$ \\
Glucocorticoids, $\mathrm{n}$ (\%) & $49(59)$ & $40(48.2)$ & $\mathrm{NS}$ \\
\hline
\end{tabular}

NS, non-significant; CVRF, cardiovascular risk factors; DM, diabetes mellitus; BMI, body mass index; SBP, systolic blood pressure; HDL-C, high density lipoprotein cholesterol.

Figure 1. Kaplan-Meier survival curve showing $96 \%$ probability of remaining alive at 5 years.

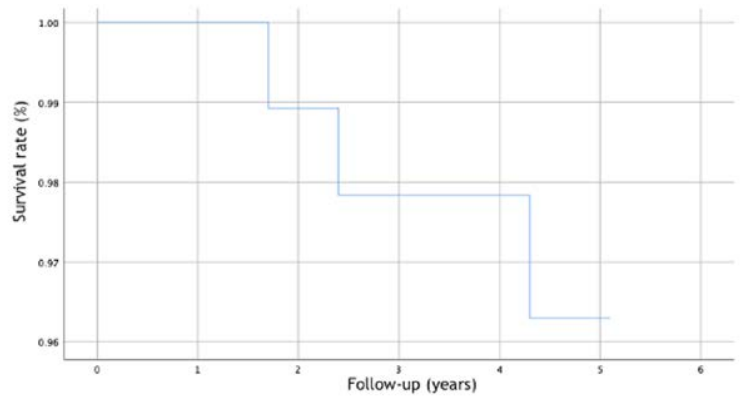

Disclosure of Interests: None declared

DOI: 10.1136/annrheumdis-2021-eular.1601

\section{AB0118 REMISSION OF ANXIETY AND DEPRESSIVE DISORDERS IMPROVES HAQ TREATMENT RESPONSE} IN RHEUMATOID ARTHRITIS PATIENTS

A. Abramkin ${ }^{1}$, T. Lisitsyna ${ }^{1}$, D. Veltishchev ${ }^{2}$, O. Seravina², O. Kovalevskaya², S. Glukhova ${ }^{1}$, E. Nasonov ${ }^{1} .^{1}$ V.A. Nasonova Research Institute of Rheumatology, Vascular Rheumatology, Moscow, Russian Federation; ${ }^{2}$ Moscow Research Institute of Psychiatry, Serbsky NMRC PN MoH, Stress Disorders, Moscow, Russian Federation

Background: Anxiety and depressive disorders (ADD) significantly affect disease activity and functional disability in rheumatoid arthritis (RA) patients. Psychopharmacotherapy (PPT) of ADD attempts to improve RA disease activity and lower progression of functional limitations.

Objectives: To determine factors associated with $\mathrm{HAQ}$ treatment response in antidepressants-treated RA-patients at five years endpoint.

Methods: 128 RA-patients (pts) were enrolled, $86 \%$ were women with a mean age of $47,4 \pm 11,3(M \pm S D)$ yrs. All patients met the full ACR/EULAR 2010 criteria for RA. Functional limitations were assessed using Health Assessment Questionnaire (HAQ), mean HAQ was $1,42 \pm 0,78$ at baseline. $69,4 \%$ RA-pts were already taking prednisone $(9[5 ; 10] \mathrm{mg} /$ day (Me $(25 \% ; 75 \%)), 84,4 \%$ - cDMARDs, $7,8 \%$ - bDMARDs (anti-TNF-a $-6,3 \%$, rituximab - 1,6\%). ADD were diagnosed by psychiatrist in $123(96,1 \%)$ of RA-pts in accordance with ICD-10 in semi-structured interview. Severity of depression and anxiety was evaluated with Montgomery-Asberg Depression Rating Scale (MADRS) and Hamilton Anxiety Rating Scale (HAM-A). RA-pts with ADD were divided into the following treatment groups: 1 - cDMARDs ( $n=39), 2$ - cDMARDs + PPT (sertraline or mianserine) $(n=43), 3$ - cDMARDs + bDMARDs $(n=32), 4$ - cDMARDs + bDMARDs + PPT (sertraline or mianserine) $(n=9)$. Biologics treatment duration varied from 1 to 6 years, antidepressants - from 6 to 96 weeks. Baseline HAQ scores were $1,39 \pm 0,75,1,42 \pm 0,9$, $1,58 \pm 0,76$ and $1,38 \pm 0,83$ in groups $1-4$, respectively. At 5 -yrs endpoint in 83 RA-pts differences between baseline and endpoint $\mathrm{HAQ}$ scores $(\triangle \mathrm{HAQ}=$ endpoint $\mathrm{HAQ}$ - baseline $\mathrm{HAQ}$ ) were assessed as $\mathrm{HAQ}$ treatment response with minimal clinically important difference (MCID) $(\triangle \mathrm{HAQ} \geq 0,22)$. $\mathrm{HAQ}$ response rates were $4,2 \%$, $65,5 \%, 47,6 \%$ and $76,7 \%$ in groups $1-4$, respectively, with the lowest response rate in group $1(p<0,0001)$. Logistic regression analysis was conducted to determine factors associated with RA remission rate.

Results: By univariate logistic regression, anxiety and depressive symptoms remission at 5-yrs endpoint, baseline $\mathrm{HAQ}$ and major depression, lower baseline age, BMI and DAS28, no minor depression and cardiovascular diseases at baseline were significantly $(p \leq 0,2)$ associated with $\mathrm{HAQ}$ treatment response (table 1$)$ These variables were subjected to multivariate stepwise logistic regression. Only remission of anxiety and depressive symptoms at 5-yrs endpoint (OR 6,6 $(95 \% \mathrm{Cl}$ $1,78-24,43), p=0,005)$, higher baseline $\mathrm{HAQ}$ (OR 2,61 (95\% $\mathrm{Cl} 1,12-6,11)$ $\mathrm{p}=0,027)$ and lower baseline BMI (OR $0,9(95 \% \mathrm{Cl} 0,85-0,96), p=0,001)$ were independently associated with $\mathrm{HAQ}$ treatment response at 5 -years follow-up.

Table 1. Factors associated with RA remission at $\mathbf{5}$ years (univariate logistic regression).

\begin{tabular}{|c|c|c|c|c|}
\hline \multirow[b]{2}{*}{ Factor } & \multirow[b]{2}{*}{$p$} & \multirow[b]{2}{*}{ OR } & \multicolumn{2}{|c|}{$95 \% \mathrm{Cl}$} \\
\hline & & & low & up \\
\hline Anxiety and depressive symptoms remission at 5-yrs endpoint & 0,007 & 5,0 & 1,561 & 16,016 \\
\hline Baseline $\mathrm{HAQ}$ & 0,01 & 2,657 & 1,264 & 5,588 \\
\hline Baseline major depression & 0,142 & 2,082 & 0,782 & 5,542 \\
\hline Baseline age & 0,098 & 0,992 & 0,982 & 1,002 \\
\hline Body mass index (BMI) & 0,048 & 0,981 & 0,963 & 1,0 \\
\hline Baseline minor depression & 0,167 & 0,563 & 0,249 & 1,273 \\
\hline Cardiovascular diseases & 0,1 & 0,417 & 0,147 & 1,183 \\
\hline Baseline DAS28 & 0,008 & 0,077 & 1,071 & 2,096 \\
\hline
\end{tabular}

Conclusion: higher $\mathrm{HAQ}$ and lower BMI at baseline and remission of anxiety and depressive symptoms at 5-yrs endpoint are independently associated with $\mathrm{HAQ}$ treatment response (MCID) at 5-years follow-up.

Disclosure of Interests: None declared

DOI: 10.1136/annrheumdis-2021-eular. 1658

\begin{tabular}{l|l}
\hline AB0119 & DISCORDANCE OF RAPID3 AND CDAI IN \\
RHEUMATOID ARTHRITIS
\end{tabular}

L. A. Ramrattan ${ }^{1}$, Z. Vaghaiwalla' ${ }^{1}$, S. Singh ${ }^{1}$, K. Ramsubeik ${ }^{1}$, M. Thway $^{1}$, G. Kaeley ${ }^{1}$ ${ }^{1}$ University of Florida, Rheumatology, Jacksonville, United States of America

Background: The Clinical Disease Activity Index (CDAI) and the Routine Assessment of Patient Index Data 3 (RAPID3) ascertain rheumatoid arthritis (RA) disease activity and inform treatment decisions. The CDAl has provider and patient components, whilst the RAPID3 only has patient driven measures. During the COVID-19 pandemic, telemedicine visits relied on RAPID3 as a clinical outcome measure and subsequently was incorporated into all clinical visits in addition to the CDAl. On an ad-hoc basis, discrepancies were noted for the disease activity level generated by these two measures. The purpose of this retrospective study was to formally analyze the relationship between these measures.

Objectives: To determine the concordance of the outcome measures RAPID3 with CDAl in patients with established RA as a quality improvement project.

Methods: This is a retrospective study of 49 patients that fulfilled the American College of Rheumatology 2010 criteria for Rheumatoid Arthritis. IRB approval was obtained. The medical records of patients seen between June to October 2020 at the rheumatology department at UF health were reviewed. Data collected included age, gender, race, number of years with RA, Rheumatoid factor (RF) and anti-citrullinated protein antibody (CCP Ab) positivity, disease modifying treatments, ESR and CRP as well as CDAl and RAPID3 scores as calculated by clinic staff. The charts were reviewed by the authors and RAPID3 scores were verified.

Table 1. Patient Population

\begin{tabular}{lc}
\hline Age & $35-90$ years \\
Race & African American-13 \\
& Caucasian-10 \\
& Hispanic-3 \\
Not Hispanic=13 & $35 / 49(71) \%$ \\
RF Positive & $37 / 49(75 \%)$ \\
CCP Ab & $32 / 49(65 \%)$ \\
Both RF and CCP Ab Positive & $14 / 48(29 \%)$ \\
Patients on monotherapy & $29 / 48(60 \%)$ \\
Patients on dual therapy & $5 / 48(10)$ \\
Patients on triple therapy & Plaquenil, methotrexate, leflunomide, etanercept, \\
Antirheumatic Drugs used & adalimumab, infliximab, tofacitinib, upadacitinib, \\
& rituximab \\
CDAI and RAPID3 Concordance & $18 / 49(37 \%)$ \\
RAPID 3 Higher than CDAl & $22 / 49(45 \%)$ \\
RAPID 3 lower than CDAI & $7 / 49(14 \%)$ \\
Incorrect Calculation of RAPID3 by & $11 / 42(26 \%)$ \\
clinic staft &
\end{tabular}

